\title{
A Ring of X-rays from the Cartwheel Galaxy
}

\author{
Ginevra Trinchieri ${ }^{1,2}$, Anna Wolter ${ }^{2}$, and Angela Iovino ${ }^{2}$ \\ 1 Max-Planck-Institut für extraterrestrische Physik, D-85748 Garching, Germany \\ 2 Osservatorio Astronomico di Brera, via Brera 28, I-20121 Milano, Italy
}

\begin{abstract}
ROSAT HRI observations of the Cartwheel galaxy indicate that there is $\mathrm{X}$-ray emission from the outer ring in connection with the strongest HII regions in the southern portion of the ring. No significant emission is detected from the nucleus or from the inner ring. This implies a strong connection between star formation activity and X-ray emission in this ring galaxy.
\end{abstract}

\section{The Cartwheel: a Spectacular Ring Galaxy}

Nearly head-on collisions between a compact galaxy and a gaseous disk are thought to be the origin of ring galaxies (Lynds \& Toomre 1976; Theys \& Spiegel 1977; Toomre 1978). The density wave produced in the collision propagates outwards from the nucleus, and sweeps up material to form a relatively symmetrical ring, where high densities can be reached and star formation phenomena induced. Evidence of high levels of star formation in rings is based on their blue optical colors, large far infrared luminosities and large luminous Hil regions (Appleton \& Struck-Marcell 1987; Fosbury \& Hawarden 1977, Higdon 1995).

One of the more spectacular and famous examples of a recently formed ring galaxy is the A0035-324 system, named "Cartwheel" because of its optical appearance with a bright, sharp outer ring linked by spiral spokes to the nucleus. An inner ring close to the core is also well studied recently with HST (Borne et al 1996; Struck et al. 1996). Many detailed observations of this object are available, from radio line and continuum, to near and far infrared, optical and $\mathrm{H} \alpha$ images and line spectroscopy. All confirm the presence of a recent starburst in the outer ring, without corresponding activity in the inner ring, nucleus or spokes, believed to be relatively devoid of gas. Most of the activity is in fact in the S-SW quadrant of the ring, where massive and luminous $\mathrm{HII}$ regions characterized by large $\mathrm{H} \alpha$ luminosities and equivalent widths are found (Higdon 1995). Both dynamical considerations and stellar evolution models suggest an age of $2-4 \times 10^{8} \mathrm{yr}$ for the starburst; the evidence of very low metallicity, with large deficiency factors in $\mathrm{O}, \mathrm{N}$ and Ne relative to the solar value, and the estimated supernova rate, almost two orders of magnitude higher than in normal galaxies, also support the view that star formation in the ring is a recent phenomenon and that gas currently forming stars was nearly primordial at the time of the impact (Fosbury \& Hawarden 1977; Higdon 1995; Marcum et al 1992). The Cartwheel is located in a tight 


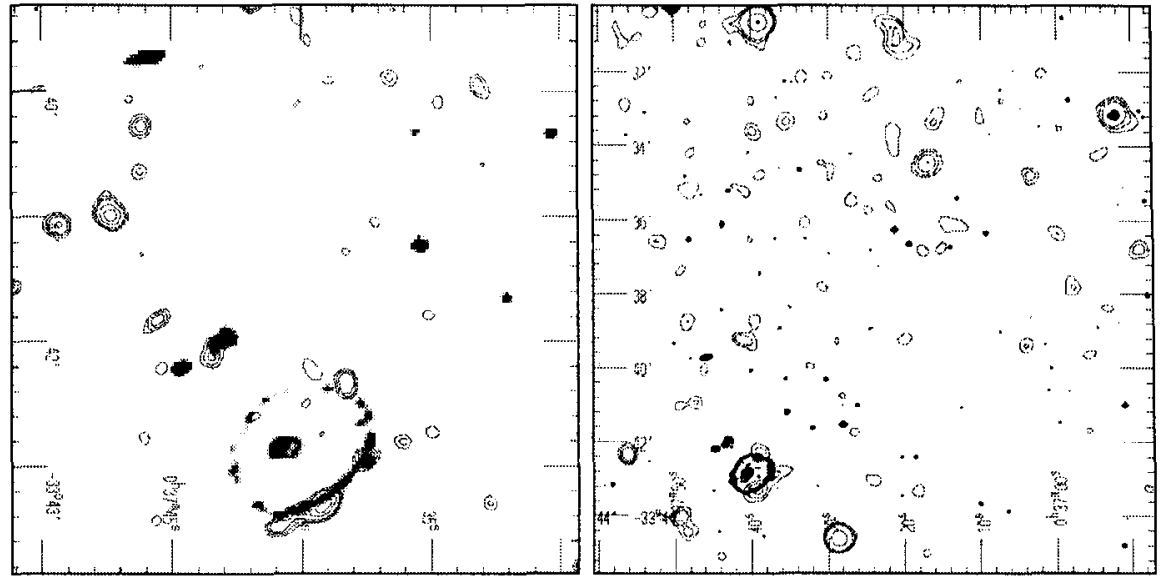

Fig. 1. Left: X-ray emission from the Cartwheel and its 3 companions superposed on the Digitized Sky Survey. The HRI data are smoothed with a $\sigma=4^{\prime \prime}$ Gaussian function. Right: In this larger field several optical counterparts coincident with $\mathrm{X}$-ray sources are visible. A $\sigma=10^{\prime \prime}$ smoothing is applied to the X-ray data.

group ( $\sim 0.2 \mathrm{Mpc}$ size) that includes three nearby companions of comparable size and magnitude, with a redshift within $400 \mathrm{~km} \mathrm{~s}^{-1}$ from one another.

All of the above indicates that X-ray observations could show both the galaxy and the group as as a source of emission, as a consequence of the star formation activity and in analogy with similar systems. As the Cartwheel is relatively large in size ( $80^{\prime \prime}$ along the major axis, corresponding to $65 \mathrm{kpc}$ at the distance of $180 \mathrm{Mpc}$ ), the outer ring can be well separated from the inner core, and the emission from the Cartwheel from that of other components of the group. We present here some preliminary results from a $\sim 60 \mathrm{ks}$ observation we obtained with the ROSAT HRI.

\section{The Cartwheel in X-rays}

ROSAT data (Fig. 1) indicate clearly that the emission comes from the outer ring, and in particular from the Southern quadrant, while the nucleus, inner ring and spokes do not emit. The coincidence of several other X-ray sources in the field with optical counterparts (see Fig. 1, right panel) ensures that the emission is really coming from the ring, and that no errors in the pointing or in the aspect solution could have determined this coincidence.

We have detected $\sim 80$ net counts above an average field background of $\sim 0.07 \mathrm{cts}^{\mathrm{arcsec}}{ }^{-2}$ from the Cartwheel. For an assumed Raymond-type spectrum with $\mathrm{kT}=1 \mathrm{keV}$ and $20 \%$ cosmic abundance, and low energy absorption corresponding to the line-of-sight column density $\mathrm{N}_{\mathrm{H}}=1.8 \times 10^{20}$ 


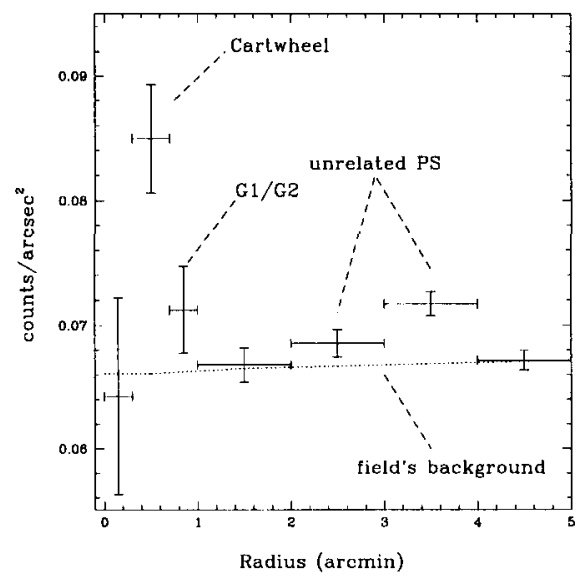

Fig. 2. Radial distribution of the total counts detected in the HRI observation $(+)$ and of the field's background, both centered on the Cartwheel.

$\mathrm{cm}^{-2}$ (Stark et al. 1992), this corresponds to a flux $\mathrm{f}_{x} \sim 5.5 \times 10^{-14} \mathrm{erg} \mathrm{cm}^{-2}$ $\mathrm{s}^{-1}$ and luminosity $\mathrm{L}_{x} \sim 2.0 \times 10^{41} \mathrm{erg} \mathrm{s}^{-1}$. The luminosity could increase to $\mathrm{L}_{x} \sim 4.0 \times 10^{41} \mathrm{erg} \mathrm{s}^{-1}$ if an intrinsic absorption of $\mathrm{N}_{\mathrm{H}}=2 \times 10^{21} \mathrm{~cm}^{-2}$ (corresponding to the reddening observed in the Hil regions), is assumed. Note that the result is virtually insensitive to the choice of $\mathrm{kT}$ and abundance.

As shown by Fig. 2, all of the emission is in an annulus at $\sim 0.3^{\prime}-1^{\prime}$ from the nucleus, and can be attributed to the outer ring of the Cartwheel (the majority) and to the two nearmost companions G1-G2 (which are probably a separate interacting pair, Higdon 1996). No emission is detected interior to the ring, or associated with the group or with the companion G3, which is now believed to be the intruder responsible for the impact with the Cartwheel (Higdon 1996). A small fraction $(\sim 1 / 10)$ of the flux attributed to the Cartwheel might be associated with a star superimposed on the outer ring, well visible in the HST image (NASA Press Release PRC96-36a, Nov. 26,1996 ) and already identified as an object with the colors of a $\mathrm{G}$ star (Marcum et al. 1992). The X-ray to optical flux ratio (estimated at $5 \times 10^{-15} \mathrm{erg}$ $\mathrm{cm}^{-2} \mathrm{~s}^{-1}$ for a $17-18 \mathrm{mag}$. object) is consistent with values of stars in this class (see nomogram in Maccacaro et al. 1988).

\section{Why X-rays on the Ring?}

We see a remarkable coincidence between $\mathrm{X}$-ray and $\mathrm{H} \alpha$ emission, indicative of a close association with the star formation phenomenon. X-ray emission is higher in connection with the brightest Hil regions while no emission is observed from the nucleus or inner ring, where no star formation enhancement is inferred from optical, radio or IR data. 
In spite of the relatively large number of young stars, coronal emission from normal stars is unlikely to account for the observed $\mathrm{L}_{X}$. Optical and infrared data infer the presence of a few $\times 10^{5} \mathrm{O}$ stars in the whole ring (Marcum et al. 1992). This implies $\lesssim 10^{7}$ O-B type stars, which would produce $<10 \%$ of the observed luminosity at most, even in the generous assumption that all emit at the highest level of $\sim 10^{33} \mathrm{erg} \mathrm{s}^{-1}$ detected from stars in our galaxy (Vaiana et al. 1981; Chlebowski et al. 1989). Galactic scale strong shocks are excluded from radio or optical observations (Higdon 1996).

It is therefore likely that the evolved stellar population, in the form of binaries, supernovae and supernova remnants, is responsible for the observed emission. Giant Hi regions and complex structures, typically coincident with peaks of $\mathrm{H} \alpha$ emission, are also found in actively star forming objects like the interacting system "The Antennae" (Read et al. 1995; Fabbiano et al. 1997) as extremely bright X-ray sources, with intrinsic luminosities up to several $\times 10^{40} \mathrm{erg} \mathrm{s}^{-1}$ and could be analogous to sources in the Cartwheel's ring.

Emission from the intergroup gas, if any, is very low, at least a factor of 10 less than other groups. The lack of emission from diffuse hot gas could be consistent with the suggestion that the this component is less dense, colder or simply less in late-type dominated groups (see Ponman et al. 1996; Mulchaey et al. 1996) or could be a further indication that most of the emission detected in these systems is due to singular phenomena (like for example shocks between interacting members like in Stephan's Quintet; Pietsch et al. 1997).

\section{References}

Appleton P.N. and Struck-Marcell C. (1987): ApJ 318, 103

Borne, K.D. et al. (1996): Science with the Hubble Telescope II, P. Benvenuti, F.D. Macchetto, E. Schreier eds. p. 239.

Chlebowski, T., Harnden, F.R., and Sciortino, S. (1989): ApJ 341, 427.

Fabbiano, G., Schweizer, F., and Mackie, G. (1977); ApJ

Fosbury, R.A. and Hawarden, T.G. (1977): MNRAS 178, 473

Higdon, J.L. (1995): ApJ 455, 524.

Higdon, J.L. (1996): ApJ 467, 241

Lynds, R. and Toomre, A. (1976): ApJ 209, 382

Marcum, P.M., Appleton, P.N. and Higdon, J. (1992): ApJ 399, 57.

Maccacaro, T. et al. (1988): ApJ 326, 680

Mulchaey J.S. et al. (1996): ApJ 456, 80

Pietsch, W. Trinchieri, G., Arp, H., and Sulentic J.W. (1997): A\&A 322, 89

Ponman, T.J., et al., (1996): MNRAS 283, 690

Read A.M., Ponman T.J. and Wolstencroft R.D. (1995): MNRAS 277, 397

Stark, A.A, et al. (1992): ApJS 79, 77

Struck, C., Appleton, P.N., Borne, K.D. and Lucas, R.A. (1996): AJ 112, 1868

Theys, J.C. and Spiegel, A.E. (1977): ApJ 212, 616

Toomre, A. (1978): IAU Symp. 79, p. 109

Vaiana, G.S. et al. (1981): ApJ 245, 163 